\title{
EVERY COUNTABLE-CODIMENSIONAL SUBSPACE OF A BARRELLED SPACE IS BARRELLED ${ }^{1}$
}

\author{
STEPHEN SAXON ${ }^{2}$ AND MARK LEVIN
}

Abstract. As indicated by the title, the main result of this paper is a straightforward generalization of the following two theorems by J. Dieudonné and by I. Amemiya and Y. Kōmura, respectively:

(i) Every finite-codimensional subspace of a barrelled space is barrelled.

(ii) Every countable-codimensional subspace of a metrizable barrelled space is barrelled.

The result strengthens two theorems by G. Köthe based on (i) and (ii), and provides examples of spaces satisf ying the hypothesis of a theorem by S. Saxon.

Introduction. N. Bourbaki [2] observed that if $E$ is a separable, infinite-dimensional Banach space, then $E$ contains a dense subspace $M$ of countably infinite codimension which is a Baire space. R. E. Edwards [4] noted that since $M$ is Baire, it is an example of a noncomplete normed space which is barrelled. Obviously, (i) and (ii) provide a plethora of such examples. It is apparently unknown whether every countable- (or even finite-) codimensional subspace of an arbitrary Baire space is Baire; (for closed subspaces the results are affirmative). In the second paper [8], which follows, the authors give topological properties other than "barrelledness" which are inherited by subspaces having the algebraic property of countable-codimensionality.

1. The notation will be that used by J. Horváth [5]. If $(E, F)$ is a dual pairing ( $E$ and $F$ not necessarily separating points) then $\sigma(E, F)$

Presented to the Society, August 30, 1968 under the title On determining barrelled subspaces of barrelled spaces and November 9, 1968; received by the editors December 13, 1968 and, in revised form, June 10, 1970.

AMS 1970 subject classifications. Primary 46A07, 47A55; Secondary 46A30, 46А 35, 46A40.

Key words and phrases. Locally convex space, barrelled space, Pták space, Mackey space with property (S), algebraic property of countable-codimensionality, strongest locally convex topology, the bipolar theorem, a perturbation theorem, Schauder basis, positive cone, closed and bounded base.

1 It has just been learned that M. Valdivia has an independent proof of this fact, to appear in Ann. Inst. Fourier (Grenoble) under the title Absolutely convex sets in barrelled spaces.

${ }^{2}$ Some of the contributions of the first-named author are included in his Florida State University dissertation which was written under the supervision of Professor C. W. McArthur . 
will denote the topology on $E$ of pointwise convergence on $F . \tau(F, E)$ will denote the topology on $F$ of uniform convergence on $\sigma(E, F)$ compact subsets of $E$. The vector space of continuous linear functionals on a locally convex space $E$ will be designated as $E^{\prime}$. A (not necessarily Hausdorff) locally convex space is said to be barrelled if every closed, balanced, convex, absorbing subset of it is a neighborhood of 0 . A locally convex space $E$ is said to be a Mackey space if it has the topology $\tau\left(E, E^{\prime}\right)$; to have property (S) if $E^{\prime}$ is $\sigma\left(E^{\prime}, E\right)$ sequentially complete. The codimension of a linear subspace $M$ of a vector space $E$ is the (algebraic) dimension of the quotient vector space $E / M$. $\omega$ will stand for the set of natural numbers.

2. To attack first the case of closed linear subspaces of countable codimension we prove the following:

LEMмA. Let $M$ be a closed linear subspace of countable codimension in a locally convex space $E$. If $E$ has property $(\mathrm{S})$, then any linear extension to $E$ of a continuous linear functional on $M$ is continuous.

Proof. Let $P=\left\{x_{n}: n \in \omega\right\}$ be a countable set such that $E$ is the linear span of $M \cup P$. Let $M_{n}$ be the linear subspace spanned by $M \cup\left\{x_{1}, \cdots, x_{n}\right\}$. Let $f$ be a continuous linear functional on $M$ and let $f_{0}$ be any linear extension of $f$ on $E$. For each $n, M$ is a closed linear subspace of finite codimension in $M_{n}$. Therefore the restriction $f_{n}$ of $f_{0}$ to $M_{n}$ is continuous. Let $g_{n}$ be any continuous extension of $f_{n}$ to $E$. An element $x$ of $E$ belongs to $M_{n}$ for some $n \in \omega$. Therefore the sequence $\left\{g_{n}(x)\right\}_{n \in \omega}$ is eventually constant and converges to $f_{0}(x)$. Since $E$ was supposed to have property (S), $f_{0}$ is a member of $E^{\prime}$, and is continuous.

Proposition. Let $E$ be a Mackey space with property (S). Let $M$ be a closed linear subspace of countable codimension in $E$. Let $N$ be any algebraic supplement of $M$. Then $N$ has the strongest locally convex topology and is a topological supplement of $M$.

Proof. Let $\pi$ be the projection of $E$ onto $N$ along $M$. Let $V$ be any absorbing, balanced, convex subset of $N$. Let $J$ be the given Mackey topology on $E$, and let $J_{0}$ be that locally convex topology on $E$ which has as a fundamental system of neighborhoods of zero all sets of the form $U \cap \epsilon \cdot(M+V)=U \cap(M+\epsilon \cdot V)$, where $U$ is a J-neighborhood of zero and $\epsilon>0$. Clearly, $J_{0}$ is stronger than $J$, so that $(E, J)^{\prime}$ $\subset\left(E, J_{0}\right)^{\prime}$. The relative topologies $\left.\Im\right|_{M}$ and $\left.\Im_{0}\right|_{M}$ are coincident on $M$. Thus $\left.f \in\left(E, J_{0}\right)^{\prime} \Rightarrow f\right|_{M} \in\left(M,\left.J_{0}\right|_{M}\right)^{\prime}=\left(M,\left.J\right|_{M}\right)^{\prime} \Rightarrow f \in(E, J)^{\prime}$ by the lemma. That is, $(E, J)^{\prime}=\left(E, J_{0}\right)^{\prime}$. But since $\mathfrak{J}$ is the Mackey topology, we now have that $J$ is stronger than $J_{0}$, and $M+V$ is a $J$-neighbor- 
hood of zero. We have shown two things, then, that $N$ has the strongest locally convex topology and that for any absorbing, balanced, convex subset $V$ of $N, \pi^{-1}(V)=M+V$ is a neighborhood of zero in $E$. That is, $\pi$ is continuous, and $N$ is a topological supplement to $M$.

Corollary A. A closed subspace $M$ of countable codimension in a barrelled space $E$ is barrelled.

Proof. A barrelled space is Mackey and has property (S), so the preceding proposition applies. $M$ is isomorphic to $E / N$, so $M$ is barrelled.

Corollary B. A countable-dimensional barrelled space must be isomorphic to the product of a vector space with the strongest locally convex topology and one with the indiscrete topology.

Proof. The closure of $\{0\}$ is a closed subspace of countable codimension.

Corollary C. A metrizable locally convex space $E$ of countably infinite dimension is not barrelled.

Proof. If $E$ were barrelled, $E$ would have the strongest locally convex topology, since $\{0\}$ is closed. Then every linear functional on $E$ would be continuous. $E$ must contain a linearly independent sequence convergent to zero. The linear functional, necessarily continuous, which assumes value 1 on every element of the sequence provides a contradiction.

3. In handling the case of dense linear subspaces of countable codimension, we utilize the following:

Lemma. Let $E$ be a locally convex space with property (S). Let $A$ be a balanced, convex, closed subset of $E$. If the linear span $\operatorname{sp}(A)$ of $A$ has countable codimension in $E$, then $\operatorname{sp}(A)$ is closed in $E$.

Proof. The proof is explicitly given for the $\boldsymbol{\aleph}_{0}$-codimensional case. Let $x_{1}$ be any element of $E$ not in $\operatorname{sp}(A)$, and let $\left\{x_{k}\right\}_{k=1}^{\infty}$ be a linearly independent sequence in $E$ such that $\operatorname{sp}\left(\left\{x_{k}\right\}\right)$ and $\operatorname{sp}(A)$ are algebraic supplements to each other. By the bipolar theorem [5, p. 192] and since $E$ is locally convex, we have $A^{00}=A$, where the (absolute) polars are taken with respect to the canonical pairing between $E$ and $E^{\prime}$. Since no nonzero scalar multiple of $x_{k}$ is in $A$, it thus follows that $A^{0}$ is unbounded at $x_{k}$ for $k=1,2, \cdots$. Let $\epsilon>0$ be given, with $\epsilon<1$. Let $A_{1}=A$ and let $A_{n}=A_{n-1}+\left\{t x_{n-1}:|t| \leqq 1\right\}$ for $n=2,3, \cdots$. Then $A_{n}^{00}=A_{n}$ for $n=1,2, \cdots$. Choose a sequence $\left\{f_{n}\right\}$ such that $f_{n}$ 
$\in\left(2^{-n} \cdot \epsilon\right) A_{n}^{0}, f_{1}\left(x_{1}\right)=2$, and $\sum_{k=1}^{n+1} f_{k}\left(x_{n+1}\right)=0$ for $n=1,2, \cdots$ This is possible since $A_{n}^{0}$ is balanced and $\left\{f\left(x_{n}\right): f \in A_{n}^{0}\right\}$ is unbounded for $n=1,2, \cdots$. Let $x \in E$. Since $A_{n}$ is absorbing in $\operatorname{sp}\left(A_{n}\right)$ and since $\bigcup_{n=1}^{\infty} \operatorname{sp}\left(A_{n}\right)=E$, there is some integer $p$ and a positive number $\delta$ such that $\delta x \in A_{p} \subset A_{p+1} \subset A_{p+2} \cdots$. Thus

$$
\begin{aligned}
\sum_{k=1}^{\infty}\left|f_{k}(x)\right| & =1 / \delta \sum_{k=1}^{\infty}\left|f_{k}(\delta x)\right|=1 / \delta\left(\sum_{k=1}^{p-1}\left|f_{k}(\delta x)\right|+\sum_{k=p}^{\infty}\left|f_{k}(\delta x)\right|\right) \\
& \leqq 1 / \delta\left(\sum_{k=1}^{p-1}\left|f_{k}(\delta x)\right|+\sum_{k=p}^{\infty} 2^{-k} \cdot \epsilon\right)<\infty .
\end{aligned}
$$

Since $E^{\prime}$ is $\sigma\left(E^{\prime}, E\right)$-sequentially complete, the linear functional $f_{c}$ defined by $f_{\epsilon}(x)=\sum_{k=1}^{\infty} f_{k}(x)$ is continuous on $E$. Furthermore, for $x \in A_{1}$,

$$
\left|f_{\epsilon}(x)\right| \leqq \sum_{k=1}^{\infty}\left|f_{k}(x)\right| \leqq \sum_{k=1}^{\infty} 2^{-k} \cdot \epsilon=\epsilon
$$

and

$$
\left|f_{\epsilon}\left(x_{n+1}\right)\right| \leqq\left|\sum_{k=1}^{n+1} f_{k}\left(x_{n+1}\right)\right|+\sum_{k=n+2}^{\infty}\left|f_{k}\left(x_{n+1}\right)\right| \leqq 0+\sum_{k=n+2}^{\infty} 2^{-k} \cdot \epsilon<\epsilon
$$

for $n=1,2, \cdots$. Also, $\left|f_{\epsilon}\left(x_{1}\right)\right| \geqq 2-\sum_{k=2}^{\infty}\left|f_{k}\left(x_{1}\right)\right|>2-\epsilon>1$. Let $g_{\epsilon}=\left(f_{\epsilon}\left(x_{1}\right)\right)^{-1} f_{\epsilon}$. Then $g_{\epsilon}\left(x_{1}\right)=1,\left|g_{\epsilon}(x)\right|<\epsilon$ for $x \in A_{1}=A$, and $\left|g_{\epsilon}\left(x_{n+1}\right)\right|$ $<\epsilon$ for $n=1,2, \cdots$. Let $\left\{\epsilon_{n}\right\}$ be a sequence such that $0<\epsilon_{n}<1$ $(n=1,2, \cdots)$ and $\epsilon_{n} \rightarrow 0$. The linear functional $h$ defined on $E$ by

$$
\begin{aligned}
h(x)=0 & \text { if } x \in A_{1}, \\
=1 & \text { if } x=x_{1}, \\
=0 & \text { if } x=x_{n+1}, \quad \text { for } n=1,2, \cdots,
\end{aligned}
$$

is the pointwise limit of a sequence $\left\{g_{\epsilon_{n}}\right\}_{n=1}^{\infty}$ of continuous linear functionals, and hence is continuous. Therefore the closure of $\operatorname{sp}(A)$ is a subset of $h^{-1}(\{0\})$ and does not contain $x_{1}$. It follows that $\operatorname{sp}(A)$ is closed.

MAIN TheOREM. Let $M$ be a countable-codimensional linear subspace of a barrelled space $E$. Then $M$ is barrelled.

Proof. Let $T$ be a barrel in $M$. Then its closure $\bar{T}$ in $E$ is a barrel in its linear $\operatorname{span} \operatorname{sp}(\bar{T})$. Since $M=\operatorname{sp}(T) \subset \operatorname{sp}(\bar{T}) \subset E$, the codimension of $\operatorname{sp}(\bar{T})$ in $E$ is countable. Hence by the lemma,

$$
\operatorname{sp}(\bar{T})=\overline{\operatorname{sp}(T)}=\bar{M},
$$


and $\bar{T}$ is a neighborhood of zero in the barrelled space $\bar{M}$, by Corollary A. It follows that $T=\bar{T} \cap M$ is a neighborhood of zero in $M$.

4. The following improves Köthe's extensions [7] of a perturbation theorem by T. Kato:

Proposition. Let $E$ be a Ptak space, $F$ a barrelled space, and $A$ a closed linear map on $D[A] \subset E$ into $F$. If the image space $R[A]$ is of countable codimension in $F$, then $A$ is open and $R[A]$ is closed in $F$. Moreover, if $F$ does not contain a complemented $\boldsymbol{\aleph}_{0}$-dimensional subspace with the strongest locally convex topology (in particular, if $F$ is metrizable) then $R[A]$ has finite codimension in $F$.

Proof. $R[A]$ is a barrelled space, so that the proof [7] of Köthe's first theorem applies, as does, in turn, our Proposition 2 (and its Corollary C).

Techniques used in S. Saxon's dissertation [9, Chapter 3] show, without use of the continuum hypothesis, that every subspace with codimension less than $2^{\aleph_{0}}$ in a Fréchet space is barrelled, and if closed, is finite-codimensional. This gives another generalization (the best possible as regards codimensionality) of Kato's theorem [6]. (It is apparently also new in Kato's original setting of Banach spaces.)

Theorem. Let $E$ be a Pták space, $F$ a Fréchet space, and $A$ a closed linear map on $D[A] \subset E$ into $F$. If the codimension of $R[A]$ is less than $2 \aleph_{0}$, then $A$ is open and $R[A]$ is closed and of finite codimension in $F$.

5. The results of this paper (as well as [1], [3], and [4]) assure the existence of barrelled (and proper) subspaces of the Banach space $l_{1}$ which contain the unit vector basis $\left\{e_{i}\right\}$ of $l_{1}$. The importance of these spaces is evident in the following:

TheоRem. Let $K$ be the positive cone of a Schauder basis $\left\{x_{i}\right\}$ in a locally convex Hausdorff space $E$ with $K-K$ barrelled. Then the following are equivalent.

(i) $\left\{x_{i}\right\}$ is a normalizable absolute (Schauder) basis.

(ii) $E$ is, by identification, a barrelled subspace of $l_{1}$ which contains $\left\{e_{i}\right\}$ as a normalization of $\left\{x_{i}\right\}$.

(iii) $K$ has a bounded base.

(iv) K has a closed and bounded base.

This theorem is Corollary 7 to Theorem 2.6 of S. Saxon [9].

6. The following examples show that sometimes dense linear subspaces of uncountable codimension in barrelled spaces are barrelled, and sometimes they are not. 
Example A. A dense linear subspace of uncountable codimension in a barrelled space which is barrelled. The linear span $L$ of a basis in a Banach space $E$ is of countable dimension. By Corollary $\mathrm{C}$ to the proposition of paragraph 2, $L$ is not barrelled. By the Main Theorem, $L$ is of uncountable codimension. There must exist an increasing sequence $\left\{L_{n}\right\}_{n \in \omega}$ of linear subspaces of $E$ such that $L_{n}$ is of uncountable codimension in $L_{n+1}$ for each $n \in \omega, L_{1}=L$ and $E=\cup_{n \in \omega} L_{n}$. Since $E$ is a Baire space, it cannot be the union of a countable collection of sets which are nowhere dense in themselves. Then some $L_{n}$ is a Baire space, and so is barrelled (cf. [2]).

EXAMPle B. A dense linear subspace of a barrelled space which has uncountable dimension and fails to be barrelled. Let $s$ be the space of real sequences with the product topology. Let $m$ be the dense linear subspace of $s$ consisting of the bounded sequences. $m$ is not barrelled with the relative topology since the barrel $\left\{\left\{x_{n}\right\}_{n \in \omega}:\left|x_{n}\right| \leqq 1\right.$ for $n \in \omega\}$ is not a neighborhood of 0 in the relativised topology. By the Main Theorem, $m$ is of uncountable codimension.

REMARK. A similar example may be obtained in $l_{2}$ by considering the subspace spanned by the Hilbert cube.

The authors are very grateful to the referee for his helpful suggestions.

\section{REFERENCES}

1. I. Amemiya and Y. Kömura, Über nict-vollständige Montelräume, Math. Ann. 177 (1968), 273-277. MR 38 \#508.

2. N. Bourbaki, Livre V: Espaces vectoriels topologiques. Chapitre 3: Espaces d'applications linéaires continues, Actualités Sci. Indust., no. 1229, Hermann, Paris, 1955, p. 3. MR 17, 1109.

3. J. Dieudonné, Sur les propriêtés de permanence de certains espaces vectoriels topologiques, Ann. Soc. Polon. Math. 25 (1952), 50-55. MR 15, 38.

4. R. E. Edwards, Functional analysis. Theory and applications, Holt, Rinehart and Winston, New York, 1965, p. 461, exercises 6.21, 6.23. MR 36 \#4308.

5. J. Horváth, Topological vector spaces and distributions. Vol. I, Addison-Wesley, Reading, Mass., 1966. MR 34 \#4863.

6. T. Kato, Perturbation theory for nullity, deficiency and other quantities of linear operators, J. Analyse Math. 6 (1958), 261-322. MR 21 \#6541.

7. G. Köthe, Die Bildräume abgeschlossener Operatoren, J. Reine Angew. Math. 232 (1968), 110-111. MR 38 \#2615.

8. M. Levin and S. Saxon, $A$ note on the inheritance of properties of locally convex spaces by subspaces of countable codimension, Proc. Amer. Math. Soc. 29 (1971), 97102.

9. S. Saxon, Basis cone base theory, Dissertation, Florida State University, Tallahassee, Fla., 1969 (unpublished).

University of Florida, Gainesville, Florida 32601

Florida State University, Tallahassee, Florida 32306 